\title{
Pseudophaeochromocytoma associated with Clozapine Therapy: a case report
}

Elevation of plasma noradrenaline levels has been reported as a consistent clinical effect of clozapine, due to its potent effect on $\alpha 2$-adrenergic receptors. ${ }^{1,2,3}$ The relationship between this elevation and clinical effectiveness remains inconclusive. Davidson et $\mathrm{al}^{1}$ concluded that the elevation was unrelated to clinical effectiveness. However, Breier et $\mathrm{al}^{2}$ reported a positive correlation between plasma noradrenaline elevation and clinical improvement in patients on clozapine treatment.

Whilst the elevation remains largely asymptomatic in many patients, there have been a few case reports of a pseudophaeochromocytoma syndrome associated with clozapine treatment. Phaeochromocytomas are catecholamine producing tumours of the sympathetic nervous system, with $95 \%$ of them occurring in the adrenal glands. ${ }^{4}$

The clinical manifestations arise from their excessive production of catecholamines. Sustained or paroxysmal hypertension has been described as the sine qua non clinical finding. ${ }^{4}$ Most patients present with hypertensive crisis. Other commonly associated features include: seizures, anxiety attacks, weight loss, and hyperglycaemia. ${ }^{4}$

Diagnostic investigations include: plasma metanephrines, as well as 24-hour urinary catecholamines and metanephrines. MRI and Meta-iodobenzylguanidine (MIBG) are useful in detecting adrenal and extra-adrenal lesions respectively.

Our patient was a 51-year-old Caucasian female, diagnosed with paranoid schizophrenia, recurrent depressive illness and generalized anxiety disorder in reference to ICD-10 criteria. The patient provided informed consent for the presentation of the case material. She was admitted into a long-stay inpatient psychiatric unit for treatment. She presented with: restricted affect, auditory hallucinations, poverty of thought content, delusions of persecution and reference, nihilistic delusions, somatic hallucinations and passivity, and impaired insight. Her medications prior to admission were: olanzapine, sertraline, enalapril and nitrazepam.

Prior to clozapine initiation, she suffered from essential hypertension which was adequately controlled with low dose enalapril 2.5mg (usual maintainance; 10-40mg). ${ }^{5}$ Her FBE and ECG were normal. Her Echocardiogram showed

Correspondence

Dr O Akinsola

email: akinsolaoa@cbdha.nshealth.ca mild concentric LVH, which was consistent with chronic hypertension. Her psychotic symptoms remained refractory to treatment despite several psychotropic trials, including varying combinations of haloperidol, thioridazine, risperidone, olanzapine, amitryptilline, prothiaden, moclobemide, fluvoxamine, and sertraline.

A few months after clozapine initiation, her blood pressure control became suboptimal and this necessitated alterations of her enalapril, as advised by specialist physicians. Blood pressure remained manageable until approximately eight years into clozapine treatment, when control again deteriorated. There were concomitant symptoms such as: worsening anxiety, weight loss and dizziness. She presented with paroxysmal hypertensive crises, with blood pressure reaching 250/120mmHg on one occasion. There was associated tachycardia, with her heart rate reaching 140 beats/minuites.

Medications during this period were: clozapine $925 \mathrm{mg}$, lamotrigine $125 \mathrm{mg}$, mirtazapine $45 \mathrm{mg}$, zopiclone $7.5 \mathrm{mg}$, and enalapril 10mg. Serial serum clozapine assays were carried, with two readings $>1110 \mu \mathrm{g} / \mathrm{L}$ (reference range 100$800 \mu \mathrm{g} / \mathrm{L})$. Her urinary catecholamines were elevated: Noradrenaline 1402nmol/d (reference level <780nmol/d), adrenaline 146nmol/L (reference level <80nmol/L). Serum Normetadrenaline was 1940pmol/L (reference level $<900 \mathrm{pmol} / \mathrm{L}$ ).

Comprehensive investigations excluded all other causes of hypertension and elevated serum and urinary catecholamines. On these grounds, the diagnosis of pseudophaeochromocytoma, associated with clozapine treatment was made

The risks versus benefits of discontinuing clozapine were considered in collaboration with the treating specialist physicians. Withdrawal of clozapine was advised. Clearly, our patient had responded poorly to previous antipsychotic trials. Her response to clozapine, although partial, had resulted in reduction of distress, and improved quality of life. On balance, it was decided to maintain her on clozapine. Urinary catecholamines remained elevated a year afterwards. Antihypertensive therapy was optimized which resulted in improved blood pressure control.

It was unclear why she had elevated serum clozapine levels when the phaeochromocytoma symptoms emerged. Nevertheless, we believe the high serum clozapine level aggravated her possibly chronically elevated, but undetected, serum catecholamines.

Our detailed literature search identified a total of six previously reported cases. A summary is shown in Table I. 


\begin{tabular}{|c|c|c|c|c|c|c|c|c|c|c|c|c|}
\hline & \multirow{2}{*}{$\begin{array}{l}\text { Case } \\
\text { no }\end{array}$} & \multirow{2}{*}{$\begin{array}{l}\text { Clozapine } \\
\text { dose } \\
\text { before VMA } \\
\text { measurement }\end{array}$} & \multirow{2}{*}{\begin{tabular}{l|} 
Duration of \\
treatment \\
before onset \\
of elevated \\
blood pressure
\end{tabular}} & \multirow{2}{*}{$\begin{array}{l}\text { Duration of } \\
\text { clozapine } \\
\text { treatment } \\
\text { (months) }\end{array}$} & \multirow{2}{*}{$\begin{array}{l}\text { Concurrent } \\
\text { medications }\end{array}$} & \multirow{2}{*}{$\begin{array}{l}\text { Heart rate } \\
\text { during } \\
\text { elevated } \\
\text { blood } \\
\text { pressure }\end{array}$} & \multicolumn{3}{|c|}{ Blood pressure } & \multicolumn{2}{|c|}{ Urinary VMA } & \multirow{2}{*}{$\begin{array}{l}\text { Serum } \\
\text { clozapine } \\
(100-800, \\
\text { ug/L }\end{array}$} \\
\hline & & & & & & & $\begin{array}{l}\text { Before } \\
\text { clozapine } \\
\text { treatment }\end{array}$ & \begin{tabular}{|l} 
During \\
clozapine \\
treatment
\end{tabular} & $\begin{array}{l}\text { After } \\
\text { clozapine } \\
\text { treatment }\end{array}$ & $\begin{array}{l}\text { During } \\
\text { clozapine } \\
\text { treatment }\end{array}$ & $\begin{array}{l}\text { Stopping } \\
\text { treatment }\end{array}$ & \\
\hline \multirow[t]{4}{*}{$\begin{array}{l}\text { Krentz } \\
\text { et ala }\end{array}$} & \begin{tabular}{|l|}
1 \\
$(27, M)$
\end{tabular} & 400 & 7 days & 2 months & $\begin{array}{l}\text { Fluoxetine } \\
20 \mathrm{mg}\end{array}$ & 110 & $n / a$ & $170 / 120$ & $n / a$ & Elevated & $\begin{array}{l}\text { VMA within } \\
\text { limits }\end{array}$ & $n / a$ \\
\hline & $\begin{array}{l}2 \\
(28, F)\end{array}$ & 700 & n/a & 12 months & $\begin{array}{l}\text { Bendrofluazide } \\
2.5 \mathrm{mg} \text { daily }\end{array}$ & 104 & $n / a$ & $143 / 112$ & n/a & Elevated & $\begin{array}{l}\text { VMA within } \\
\text { limits after } \\
\text { Treatment } \\
\text { stopped }\end{array}$ & $n / a$ \\
\hline & $\begin{array}{l}3 \\
(38, M)\end{array}$ & 900 & n/a & 18 months & $\begin{array}{l}\text { Sulpiride } 600 \mathrm{mg} \\
\text { Venlafaxine } 150 \mathrm{mg} \\
\text { Metformin } 500 \mathrm{mgbd}\end{array}$ & 130 & n/a & $156 / 100$ & n/a & Elevated & $\begin{array}{l}\text { Clozapine } \\
\text { not stopped }\end{array}$ & $n / a$ \\
\hline & \begin{tabular}{|l}
4 \\
$(22, M)$
\end{tabular} & 600 & n/a & 3 months & $\begin{array}{l}\text { Sulpiride 200mg } \\
\text { Paroxetine 50mg }\end{array}$ & n/a & $120 / 80$ & $180 / 120$ & n/a & Elevated & $\begin{array}{l}\text { Clozapine } \\
\text { not stopped }\end{array}$ & n/a \\
\hline $\begin{array}{l}\dot{L} \\
\text { et al? }\end{array}$ & $\begin{array}{l}5 \\
(27, M)\end{array}$ & 300 & n/a & 2 months & $\begin{array}{l}\text { Propanol 10mg tds } \\
\text { Amlodipine } 5 \mathrm{mg}\end{array}$ & $n / a$ & $110 / 70$ & $146 / 106$ & $110 / 70$ & $\begin{array}{l}\text { Elevated } \\
\text { limits after } \\
\text { treatment } \\
\text { stopped }\end{array}$ & VMA within & $n / a$ \\
\hline $\begin{array}{l}\text { Prasad \& } \\
\text { Kennedy }\end{array}$ & $\begin{array}{l}6 \\
(44, M)\end{array}$ & 300 & 5 days & 3 months & $\begin{array}{l}\text { Lofepramine } 210 \mathrm{mg} \\
\text { Venlafaxine } 225 \mathrm{mg} \\
\text { Citalopram 20mg } \\
\text { Amlodipine } 5 \mathrm{mg}\end{array}$ & 100 & $130 / 90$ & \begin{tabular}{|l|}
$150 / 100$ \\
Systolic range \\
$(170-140)$ \\
Diastolic range \\
$(100-115)$
\end{tabular} & $120 / 70$ & Elevated & $\begin{array}{l}\text { VMA within } \\
\text { limits, 6/52 } \\
\text { after } \\
\text { stopping } \\
\text { treatment }\end{array}$ & n/a \\
\hline
\end{tabular}

There were five male subjects and one female subject. ${ }^{6,7,8}$ Their ages ranged from $22-44$ years. All patients had a diagnosis of schizophrenia, with various combinations of refractory positive and negative psychotic symptoms, as well as several failed antipsychotic trials prior to the initiation of clozapine. Dosages ranged from 300 - 900mg daily, and the duration of treatment varied from $2-18$ months. The duration of clozapine treatment before the onset of elevated blood pressure was unavailable in 4 of the reported cases. All subjects had elevated urinary catecholamines and hypertension without alternative explanation. Obesity, profuse sweating, and tachycardia were reported in 4 cases.

Clozapine was discontinued in 4 cases, with a consequent normalization of blood pressure and urinary catecholamines shortly afterwards.

Given the potentially significant morbidity associated with this syndrome, especially in undiagnosed cases, we recommend, early urinary catecholamine screening for patients who develop hypertension, or other typical symptoms after clozapine initiation. Clinical guidelines for early diagnosis and management could be developed by clozapine manufacturers internationally.

O Akinsola, K Ong

Victorian Institute of Forensic Mental Health, Thomas Embling Hospital, Fairfield, Victoria, Australia

\section{References}

1. Davidson M, Kahn RS, Stern RG, Hirschowitz J, Apter S, Knott P, Davis KL. Treatment with clozapine and its effect of plasma homovanillic acid and norepinephrine concentrations in schizophrenia. Psychiatry Research 1993; 46: 151-163.

2. Breier A, Buchanan RW, Waltrip RW, Listwak S, Holmes C, Goldstein DS. The effect of clozapine on plasma norepinephrine: relationship to clinical efficacy. Neuropsychopharmacology 1994; 10: 1-7.

3. Pickar D, Owen RR, Litman RE, Konicki E, Gutierrez R, Rapaport MH. Clinical and biologic response to clozapine in patients with schizophrenia: Cross-over comparison with fluphenazine. Arch Gen Psychiatry; 49: 345-353.

4. Walther MM, Keiser HR, Linehan WM. Phaeochromocytoma: evaluation, diagnosis, and treatment. World J Urology 1999; 17: 35-39.

5. MIMS. MIMS Australia; issue number 1; 2008.

6. Krentz AJ, Mikhail S, Cantrell P, Hill GM. Pseudophaeochromocytoma syndrome associated with clozapine. BMJ 2001; 322: 1213.

7. Li JKY, Yeung VTF, Leung CM, Chow CC, Ko GTC, So WY, Cockram CS. Clozapine: a mimicry of phaeochromocytoma. Aust N Z J Psychiatry 1997; 31: 889-891.

8. Prasad SE, Kennedy HG. Pseudophaeochromocytoma associated with clozapine treatment. Ir J Psych Med 2003; 20(4): 132-134. 